\title{
A Broadband Doherty Power Amplifier With Hybrid Class-EFJ Mode
}

\author{
Zhiwei Zhang, Zhiqun Cheng, Hang Li, Huajie Ke, and Y. Jay Guo, Fellow, IEEE
}

\begin{abstract}
This paper proposes a method that employs novel hybrid continuous class-EFJ power amplifiers (PAs) as carrier PA to design a broadband high-efficiency Doherty power amplifier (DPA). Bandwidth characteristic of the proposed DPA is analyzed in detail. By proper selection of related parameter values, up to $78 \%$ fabrication bandwidth can be obtained. Post-harmonic tuning network is applied to improve the bandwidth and enhance the efficiency. Then, a closed design process is presented to design broadband DPA based on derived theories. For validation, a broadband DPA operating in 1.2-2.8 GHz is designed and fabricated. Measurements illustrate that the DPA can deliver saturated output power between $43.7 \mathrm{dBm}$ and $44.1 \mathrm{dBm}$ in 1.2-2.8 GHz, and the saturated drain efficiency from $60.5 \%$ to 74.2\% is achieved. Moreover, drain efficiency is $48.1 \%-57.6 \%$ at the $6 \mathrm{~dB}$ power back-off. Compared with conventional DPAs, the proposed DPA exhibits superior performance of bandwidth characteristics and power back-off efficiency over a wide bandwidth.
\end{abstract}

Index Terms-Broadband, Class EFJ, Doherty power amplifier, high-efficiency.

\section{INTRODUCTION}

$\mathrm{W}$ ITH the rapid development of the wireless communication, the amount of information transmission is increased remarkably. In order to meet such data growth, signals with large bandwidth and high peak-to-average power ratio (PAPR) are generally used [1], [2]. In order to satisfy such requirements, the power amplifier (PA) has to be developed to amplify such signals. It means that PAs should have the ability to improve the efficiency at the output back-off (OBO) level in a large bandwidth. Some amplifier architectures have been proposed to amplify signals with high PAPR, such as Doherty [3], out-phasing [4], envelope tracking [5], and envelope elimination and restoration. Due to the simple structure and low cost, Doherty PAs (DPAs) are widely used in practical base stations. However, conventional DPAs have some inherent

Manuscript received 07 August, 2020; revised 10 September, 2020; revised 15 September, 2020; accepted 20 September, 2020. Date of publication xxxx; date of current version xxxxx. This work was partly supported by National Natural Science Foundation of China (Grant 61871169, Grant 91938201 and Grant 62071163) and the Natural Science Foundation of Zhejiang Provincial (Grant LZ20F010004). (Corresponding author: Zhiqun Cheng.)

Zhiwei Zhang, Zhiqun Cheng, Hang Li, and Huajie Ke are with School of Electronics and Information, Hangzhou Dianzi University, Hangzhou 310018 China (e-mail: 2361051379@qq.com; zhiqun@hdu.edu.cn; hangli@hdu.edu.cn; khj@hdu.edu.cn;).

Y. J. Guo is with the Global Big Data Technologies Center, University of Technology Sydney, Ultimo, NSW 2007, Australia (e-mail: Jay.Guo@uts.edu.au). disadvantages, e.g., narrow bandwidth and only $6 \mathrm{~dB}$ OBO range [6], [7], which still deserve further research for improvement.

There are several factors that hinder DPAs broadband, including $\lambda / 4$ transmission line, offset line, output matching networks (OMNs) and package parasitic parameters of devices [8]-[11]. For these limitations, some solutions have been proposed to increase the bandwidth of DPAs [12]-[19]. In [12] and [13], integrated compensating reactance is used in the peaking amplifier output to improve the back-off efficiency in a large bandwidth. Post-matching topology is presented to reduce the impedance transformation ratio [14]-[17]. Symmetrical DPAs with complex load impedances is demonstrated to obtain better load modulation in a wide bandwidth [18], [19]. In order to extend the OBO range, many methods have also been proposed [20]-[22]. In [20] and [21], the multiway is applied to extend OBO through using multiple peaking PA branches. The asymmetrical topology is used to make peak PA with larger saturated current than carrier PA, thus, increasing OBO [22]. All the above methods leverage the traditional class AB PAs as carrier PAs.

Recently, extended high efficiency operation modes PAs are exploited as carrier PAs to design broadband high-efficiency DPAs [23]-[25]. Although the bandwidth of DPAs is widened, the real part of the fundamental load impedance would decrease with frequency deviation from the center point [23]. As a result, the OBO drain efficiency would decline at both lower and higher operating frequency points. In [24] and [25], the carrier amplifier operates in continuous class-J at the OBO level, which can provide a fixed real part of the fundamental load impedance to maintain high-efficiency in a large bandwidth. Impedance conversion is needed to obtain fixed real part of the fundamental load impedance, while the required impedance conversion leads to the increase in the complexity of design.

A class-EFJ PA has been proposed in our previous work [26], which can effectively combine high-efficiency of class-EF PAs with large bandwidth of continuous class-J PAs at specific conditions, thereby being suitable for wideband and high efficiency purposes. The load impedance of class-EFJ PAs not only has variable imaginary part like the conventional continuous PAs, but also has inconsistent real part. Thus, such load impedance characteristics of class-EFJ PAs are highly applicable to DPAs' load modulation applications. In other words, the impedance conversion used in [24] and [25] to maintain fixed resistance can be negligible due to the load impedance characteristics of class-EFJ PAs. 
In this paper, we study continuous class-EFJ PAs in the DPAs' architecture to simplify the design process with larger bandwidth. The class-EFJ PA is used as the carrier PA of DPA. With the load impedance characteristics of the continuous class-EFJ PAs, the impedance conversion used in continuous modes DPAs to maintain fixed resistance can be simplified. The design parameters and bandwidth characteristics are derived in detail for DPAs. Up to 78\% fabrication bandwidth can be obtained when class-EFJ PA is exploited as the carrier PA. Furthermore, a closed design process is presented for broadband high efficiency DPAs with the continuous class-EFJ PAs, which greatly enhances the drain efficiency at the OBO level. A high efficiency DPA with $78 \%$ fabrication bandwidth is designed and fabricated. Compared with traditional DPAs, the proposed DPA effectively enhances fabrication bandwidth, over which the drain efficiency at the OBO level is close to that of state-of-the-art.

The structure of this paper is as follows. The theories of conventional DPAs are analyzed in Section II. Then, the theories of the proposed DPA applying continuous EFJ are derived in Section III. The closed design method and process of the broadband high-efficiency DPA are detailed in Section IV. In Section $\mathrm{V}$, simulations and measurements are given to demonstrate the performance of the proposed one, before concluding the paper in Section VI.

\section{ANALYSIS OF DPAS THEORIES}

The traditional DPA includes a carrier PA branch and a peak PA branch. At the combiner, there is a load of $R_{\mathrm{L}} / 2$. A transistor can be equivalent to an ideal current source. The equivalent traditional DPA topology is shown in Fig. 1(a). There is an impedance converter line in the carrier PA branch as shown in Fig. 1(a) [3]. $Z_{C}, Z_{\mathrm{P}}$ are the load impedances of devices for carrier and peak PAs, respectively. According to the principle of load modulation, several impedances are as follows:

$$
\begin{aligned}
& Z_{\mathrm{C} 1, \mathrm{SAT}}=(1+\beta) \cdot R_{L} \\
& Z_{\mathrm{C} 1, \mathrm{BACK}}=R_{L} \\
& Z_{\mathrm{P} 1, \mathrm{SAT}}=(1+1 / \beta) \cdot R_{L} \\
& Z_{\mathrm{P} 1, \mathrm{BACK}}=\infty,
\end{aligned}
$$

where $Z_{\mathrm{C} 1, \mathrm{SAT}}$ and $Z_{\mathrm{C} 1 \mathrm{BACK}}$ refer to $Z_{\mathrm{C} 1}$ at the saturation and power back-off levels, respectively. Similarly, $Z_{\mathrm{P} 1, \mathrm{SAT}}$ and $Z_{\mathrm{P} 1, \mathrm{BACK}}$ refer to $Z_{\mathrm{P} 1}$ at the saturation and power back-off levels, respectively. $Z_{\mathrm{C} 1}$ and $Z_{\mathrm{P} 1}$ are the load impedances of carrier and peak PAs, respectively. Similarly, $Z_{\mathrm{C}}$ and $Z_{\mathrm{P}}$ represent the load impedances of carrier and peak PA transistors, respectively. $\beta$ represents the current ratio of peak PA branch to carrier PA branch at the saturation level.

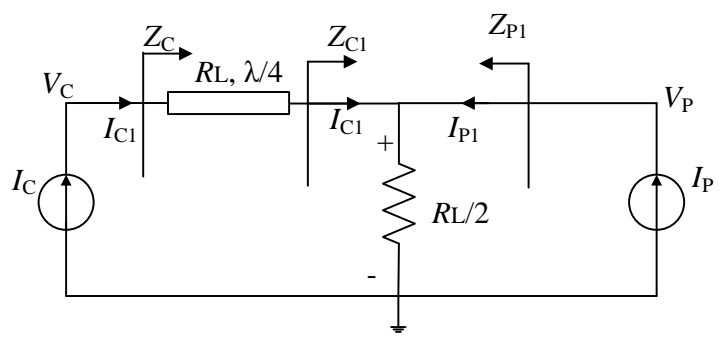

(a)

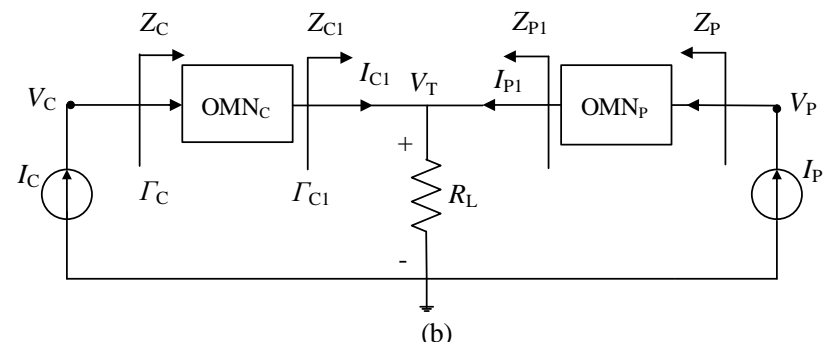

Fig. 1. DPA topology (a) Conventional DPA topology (b) Replaced topology.

Fig. 1(b) shows a more generalized topology of DPAs, where DPAs include a carrier PA branch and a peak PA branch [25]. $\mathrm{OMN}_{\mathrm{C}}$ and $\mathrm{OMN}_{\mathrm{P}}$ refer to output matching networks OMNs in the carrier PA branch and peak PA branch, respectively. At the combiner, there is a load of $R_{\mathrm{L}}$.

Assuming that $\mathrm{OMN}_{\mathrm{C}}$ is a lossless network structure, its generalized scatter parameters can be expressed as

$$
\left[\begin{array}{ll}
S_{11} & S_{12} \\
S_{21} & S_{22}
\end{array}\right]=\left[\begin{array}{cc}
0 & e^{j \theta_{C}} \\
e^{j \theta_{\mathrm{C}}} & 0
\end{array}\right]
$$

where $\theta_{\mathrm{C}}$ represents the phase delay of the network. Generally, $\theta_{\mathrm{C}}$ depends on the working frequency, and changes in linear and periodical with frequency. Here, we assume that one period is from $-\pi$ to $\pi$. Hence, during one period, $\theta_{\mathrm{C}}$ decreases from $\pi$ to $-\pi$ as the operating frequency increases. The traditional DPAs topology shown in Fig. 1(a) is consistent with the topology shown in Fig. 1(b) when $\theta_{\mathrm{C}}$ is equal to $\pi / 4$.

As shown in Fig. $1, \Gamma_{\mathrm{Cl}}$ is the reflection coefficient seen from $\mathrm{OMN}_{\mathrm{C}}$ to the carrier PA load $Z_{\mathrm{Cl}}$, and $\Gamma_{\mathrm{C}}$ is the reflection coefficient seen from carrier device into $O \mathrm{MN}_{\mathrm{C}}$. Then, we have

$$
\Gamma_{\mathrm{C}}=S_{11}+\frac{S_{12} \cdot S_{21} \cdot \Gamma_{C 1}}{1-S_{21} \cdot \Gamma_{C 1}} .
$$

Substituting (5) into (6), we can rewrite (6) as

$$
\begin{aligned}
& \Gamma_{\mathrm{C}}=\Gamma_{\mathrm{C} 1} \cdot e^{j 2 \theta_{C}} \\
& \Gamma_{\mathrm{C} 1}=\frac{Z_{\mathrm{C} 1}-(1+\beta) \cdot R_{\mathrm{L}}}{Z_{\mathrm{C} 1}+(1+\beta) \cdot R_{\mathrm{L}}} .
\end{aligned}
$$

At the saturation, combining (1), (7) and (8) can obtain

$$
\Gamma_{\mathrm{C}, \mathrm{SAT}}=\Gamma_{\mathrm{C} 1, \mathrm{SAT}}=0,
$$

where $\Gamma_{\mathrm{C} 1, \mathrm{SAT}}$ and $\Gamma_{\mathrm{C}, \mathrm{SAT}}$ represent $\Gamma_{\mathrm{C} 1}$ and $\Gamma_{\mathrm{C}}$ at the saturation power level, respectively. Equation (9) shows that the load impedance of the carrier transistor $Z_{\mathrm{C}}$ remains at $R_{\text {opt }}$ over the entire operating frequency band.

At the OBO level, the load impedance of carrier PA, $Z_{\mathrm{Cl} \text {, BACK }}$ becomes $R_{\mathrm{L}}$. Then, (8) can be calculated as

$$
\Gamma_{\mathrm{C} 1, \mathrm{BACK}}=\frac{R_{L}-(1+\beta) \cdot R_{L}}{R_{L}+(1+\beta) \cdot R_{L}}=-\frac{\beta}{2+\beta},
$$

where $\Gamma_{\mathrm{C} 1, \mathrm{BACK}}$ refers to $\Gamma_{\mathrm{C} 1}$ at the $\mathrm{OBO}$ power level.

Combining (5), (6) and (10), the reflection coefficient, $\Gamma_{\mathrm{C}, \mathrm{BACK}}$, at the $\mathrm{OBO}$ power level can be derived as

$$
\Gamma_{\mathrm{C}, \mathrm{BACK}}=\Gamma_{\mathrm{C} 1, \mathrm{BACK}} \cdot e^{j 2 \theta_{C}}=-\frac{\beta}{2+\beta} \cdot e^{j 2 \theta_{C}} .
$$

Equation (11) reveals that when PA operating at the back-off power level, the load impedance of the carrier PA, $Z_{\mathrm{C}, \mathrm{BACK}}$ is not a constant but closely related to $\theta_{\mathrm{C}}$. The $\theta_{\mathrm{C}}$ depends on the working frequency. It is obvious that $Z_{\mathrm{C}, \mathrm{BACK}}$ locates on the equal reflection. Specifically, $\beta$ equals 1 for a symmetrical DPA. Then, it can be plotted in smith chart shown in Fig. 2. 


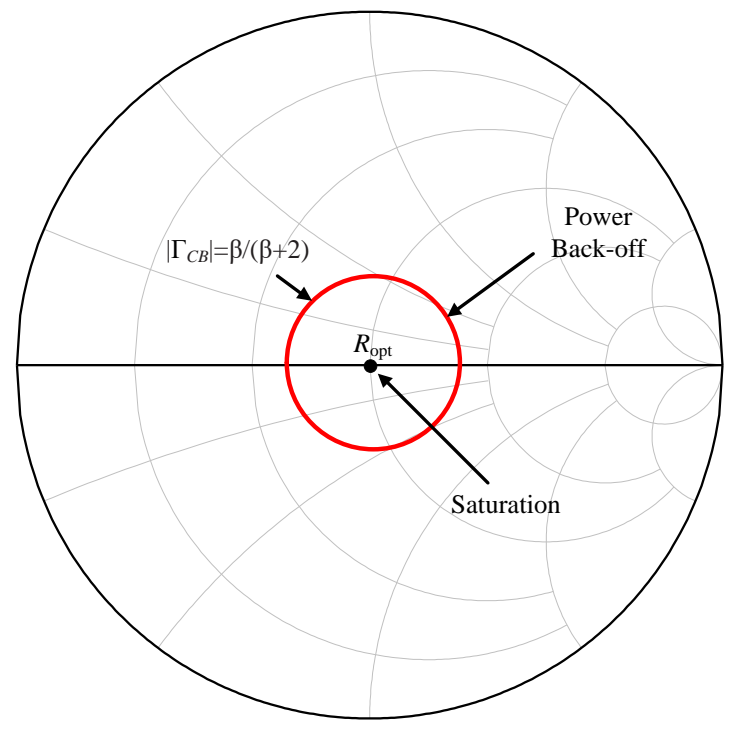

Fig. 2. The reflection coefficient $\Gamma_{\mathrm{C}}$ versus frequency.

As shown in Fig. 2, the load impedance of carrier transistor lies on a circle of constant reflection coefficient, which changes with the operating frequency at the OBO stage. Nevertheless, the load impedance of carrier PA is a constant $\left(R_{\mathrm{opt}}\right)$ in saturation, which shows that it is difficult to design a PA to satisfy the two impedance conditions at the same time. In next section, we will introduce a PA of which impedance is suitable for the impedance condition of carrier amplifier at the $\mathrm{OBO}$ stage.

\section{Theory Analysis of The Proposed DPA}

As discussed in Section II, the impedance condition of carrier PA prevents the realization of broadband DPAs. In this section, continuous class-EFJ PA is introduced to the DPA as its carrier PA. The analysis on Doherty with continuous class-EFJ PA in this paper is based on the ideal peak power amplifier branch. The fundamental impedance $Z_{f 0}$ of continuous class-EFJ PAs can be obtained from [26]

$$
Z_{f_{0}}=(1+\gamma j) * R^{*}
$$

where $\gamma$ is a coefficient taking a value from -1 to $1 . R^{*}$ is the load impedance. The available value range of $R^{*}$ is located in between $15.89 \Omega$ and $46.29 \Omega$ for the CGH40010F GaN transistor.

Compared with the traditional continuous mode class-J PA, the impedance space of class-EFJ has better flexibility, that is $R_{\mathrm{OPT}}$ in class-J is a fixed value, and $R^{*}$ in class-EFJ is an impedance space. In practical design, the appropriate value can be chosen according to different needs.

As discussed in the Section II, the load impedance of carrier PA at the OBO level is located as a constant reflection coefficient circle shown in Fig. 3. For comparison, the impedance of the continuous class-EFJ is also shown in Fig. 3. In this way, the overlapping area between the equal reflection coefficient circle and the available load impedance of the classEFJ can be clearly displayed. Seen from Fig. 3, the load impedance of class-J has only an intersection with the circle of constant reflection coefficient. This indicates that impedance conversion has to be adopted to keep the load impedance $Z_{\mathrm{C}}$ a fixed value. For class-EFJ, the situation is very different. The impedance of the continuous class-EFJ covers almost the entire iso-reflection circle. Therefore, the carrier amplifier working in the continuous class-EFJ can be more easily implemented, which simplifies the impedance process, comparing with the continuous class-J previously reported in [24]. Moreover, it can expand Doherty's bandwidth due to the extensive impedance overlap between class-EFJ and equal reflection coefficient circles.

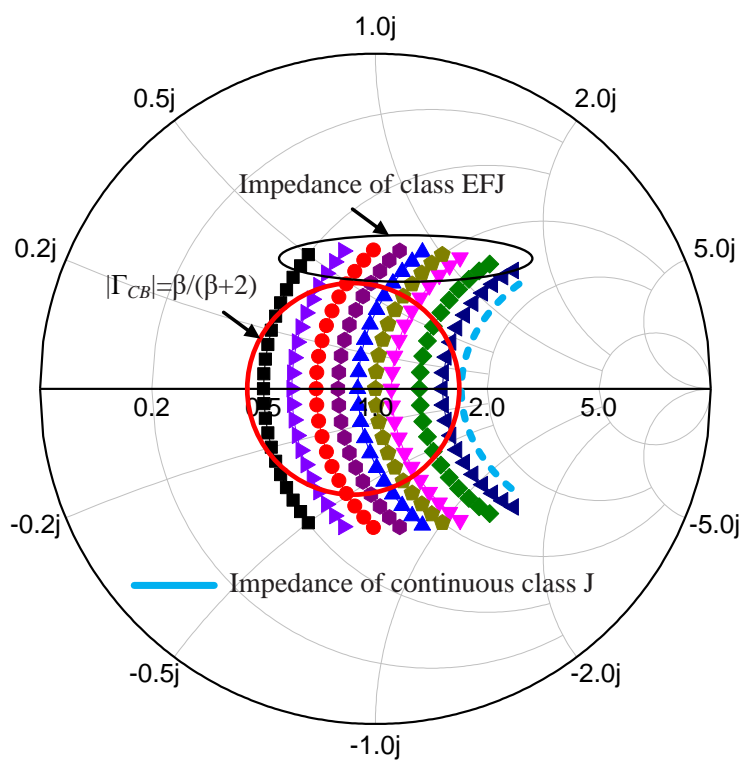

Fig. 3. Impedance of class-EFJ and continuous mode class-J.

We define the reflection coefficient corresponding to the load impedance of the continuous class-EFJ, $\Gamma_{\mathrm{EFJ}}$, as

$$
\Gamma_{E F J}=\frac{Z_{E F J}-R_{o p t}}{Z_{E F J}+R_{o p t}}
$$

Setting the condition of carrier PA to be class-EFJ at the OBO level, we have

which can be rewritten as

$$
\Gamma_{\mathrm{EFJ}}=\Gamma_{\mathrm{C}, \mathrm{BACK}},
$$

$$
\begin{aligned}
& \left|\Gamma_{\mathrm{C}, \mathrm{BACK}}\right|=\left|\Gamma_{\mathrm{EFJ}}\right| \\
& \left|\theta_{\mathrm{C}, \mathrm{BACK}}\right|=\left|\theta_{\mathrm{EFJ}}\right|,
\end{aligned}
$$

where $\theta_{\mathrm{C}, \mathrm{BACK}}$ and $\theta_{\mathrm{EFJ}}$ represent the phase of $\Gamma_{\mathrm{C}, \mathrm{BACK}}$ and $\Gamma_{\mathrm{EFJ}}$, respectively.

Substituting (11)-(13) to (15) and (16), we have

$$
\begin{aligned}
& \left(\frac{\beta}{2+\beta}\right)^{2}=\frac{R^{* 2}-R_{o p t}^{2}+\gamma^{2} \cdot R^{* 2}}{\left(R^{*}+R_{o p t}\right)^{2}+\gamma^{2} \cdot R^{* 2}} \\
& \tan 2 \theta=\frac{2 \gamma R^{*} R_{o p t}}{R^{* 2}-R_{o p t}^{2}+\gamma^{2} \cdot R^{* 2}},
\end{aligned}
$$

where

$$
\theta=\frac{\pi}{2} \frac{f}{f_{0}}
$$

Combing (18) and (19), fractional bandwidth versus $\gamma$ and $R^{*}$ can be plotted in Fig. 4. 


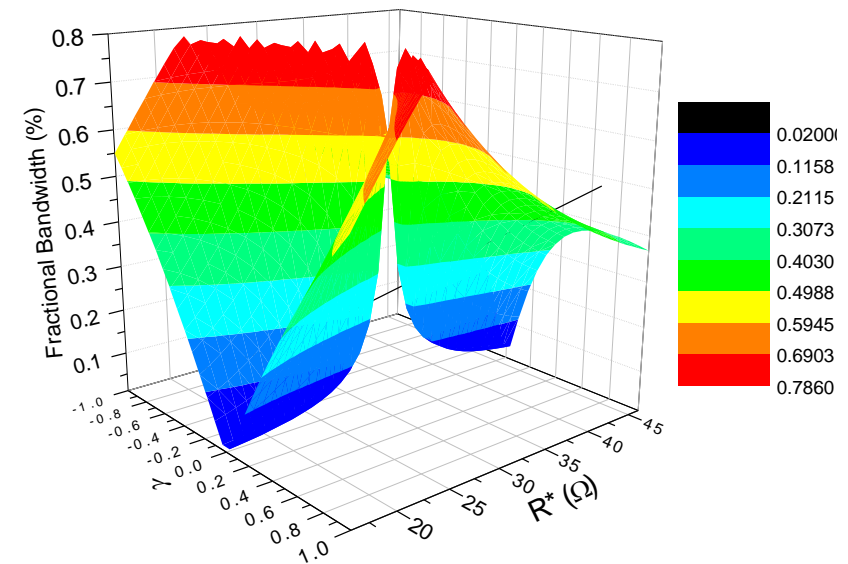

Fig. 4. Fractional bandwidth versus $\gamma$ and $R^{*}$.

From Fig. 4, different combinations of parameter values can be obtained for different relative bandwidths. In practical design, the optimal value can be chosen according to different needs. For example, when $\gamma$ and $R^{*}$ are set to -0.74 and $25 \Omega$ respectively, the maximum relative bandwidth is about $78 \%$.

Similarly, the relationship between current ration $\beta, \gamma$ and $R^{*}$ can be plotted in Fig. 5 by using (17). As shown in Fig. 5, the current ratio $\beta$ can be determined by $\gamma$ and $R^{*}$ got from Fig. 4 . For example, when $\gamma$ is -0.74 and $R^{*}$ is $25 \Omega$, with maximum relative bandwidth of $78 \%$, the current ration $\beta$ should be set as 1.16. Also, the current ratio $\beta$ has an influence on the relative bandwidth of DPAs, because different $\beta$ requires different $\gamma$ and $R^{*}$. At the same time, the relative bandwidth is determined by different $\gamma$ and $R^{*}$.

Particularly when $\gamma, R^{*}$ and $\beta$ are fixed to be $\pm 0.74,25 \Omega$ and 1.16 respectively, the bandwidth characteristics of DPAs at the OBO level are displayed in Fig. 6. It indicates that the proposed DPA can maintain high drain efficiency over a wider frequency band, compared with traditional DPAs [19]. It illustrates that the application of class-EFJ PA effectively improves the bandwidth characteristics of traditional DPAs. Next, in order to design the broadband high efficiency DPA using EFJ PAs, we derive the $A B C D$ transmission matrix of the $(\mathrm{OMN})_{\mathrm{C}}$ and $(\mathrm{OMN})_{\mathrm{P}}$.

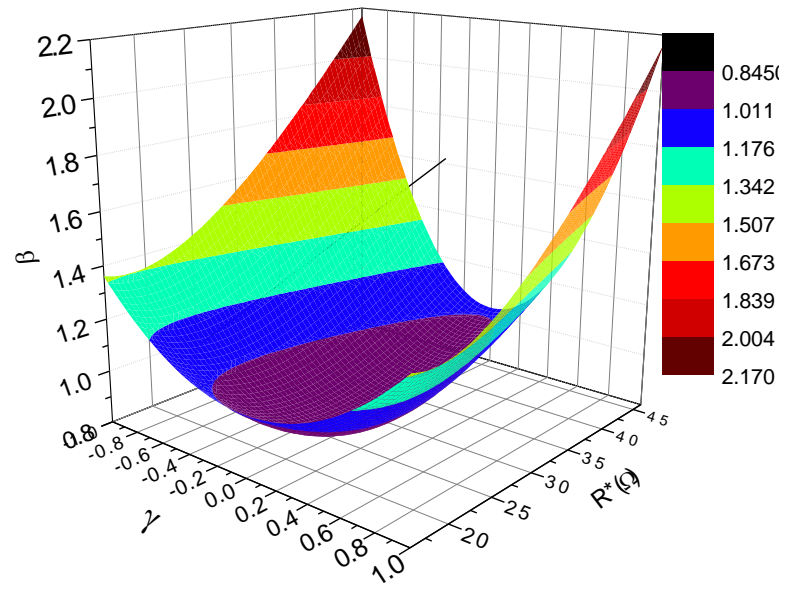

Fig. 5. Current ration $\beta$ versus $\gamma$ and $R^{*}$.

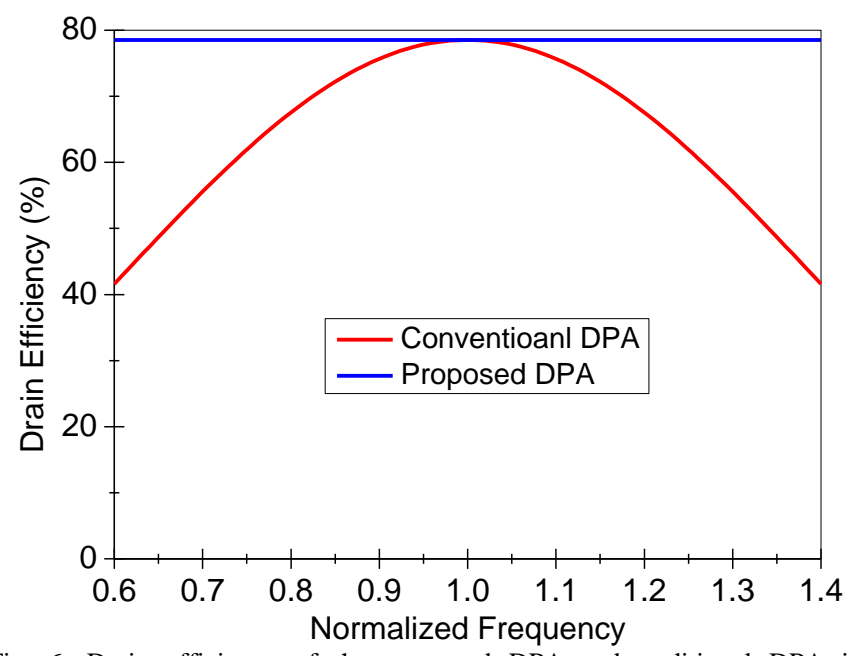

Fig. 6. Drain efficiency of the presented DPA and traditional DPA in normalized frequency of 0.6-1.4 at the OBO stage.

Fig. 7 displays impedance transformation of the DPA at the different power levels. The load impedance at the combiner $Z_{\mathrm{L}}$ is defined as $R_{\mathrm{L}} \cdot\left(1+j X_{\mathrm{L}}\right)$, where $X_{\mathrm{L}}$ refers to the normalized reactance of the $R_{\mathrm{L}}$ and is given by [27]

$$
X_{\mathrm{L}}=\mp \sqrt{\left(\alpha_{1}-2\right)\left(2 \alpha_{1}-1\right) / \alpha_{1}} \text {, }
$$

where $\alpha_{1}$ is a coefficient. In this paper, as the load impedance of carrier $\mathrm{PA}$ is $Z_{\mathrm{EFJ}}$ rather than $Z_{\mathrm{OPT}}, \alpha_{1}$ is defined as

$$
\alpha_{1}=\frac{\alpha R_{\mathrm{OPT}}}{R^{*}},
$$

where $\alpha$ represents the ratio of load impedance for carrier PA at the OBO stage to that at the saturation level.

Then, the OBO range can be derived by

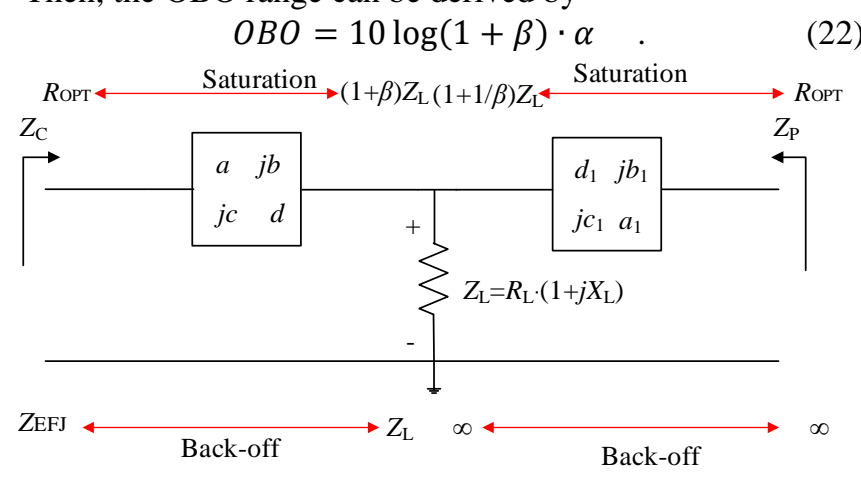

Fig. 7. Impedance conversion of the proposed DPA.

As shown in Fig. 7, in saturation, the impedance at the combiner seen from carrier branch is $(1+\beta) Z_{\mathrm{L}}$, and the load impedance $Z_{\mathrm{C}}$ is $R_{\mathrm{OPT}}$. The $(1+\beta) Z_{\mathrm{L}}$ is transformed to $R_{\mathrm{OPT}}$ by using the $O M N_{C}$. Similarly, at the OBO stage, the impedance at the combiner seen from carrier branch is $Z_{\mathrm{L}}$, and the load impedance $Z_{\mathrm{C}}$ is $Z_{\mathrm{EFJ}}$. The $Z_{\mathrm{L}}$ is also transformed to $Z_{\mathrm{EFJ}}$ by using the $\mathrm{OMN}_{\mathrm{C}}$. It is noted that regardless of $R_{\mathrm{OPT}}$ at the saturation stage or $Z_{\mathrm{EFJ}}$ at the OBO stage, it always falls within the impedance range of the class-EFJ PAs. This reduces the impact of impedance variation of the designed $\mathrm{OMN}_{\mathrm{C}}$ on the performance of the DPA due to frequency offset. Regarding the peak PA branch, the impedance at the combiner seen from peak branch is $(1+1 / \beta) Z_{\mathrm{L}}$, and the load impedance $Z_{\mathrm{P}}$ of peak transistor is $R_{\mathrm{OPT}}$ in saturation. The $(1+1 / \beta) Z_{\mathrm{L}}$ is transformed to 
$R_{\text {OPT }}$ by using the OMNp. At the OBO level, the peak transistor is off, since the impedance $Z_{\mathrm{P}}$ is infinite. Accordingly, the impedance at the combiner seen from peak brand is also infinite. This impedance conversion is also achieved by using the OMNP.

Based on the impedance conversion in Fig. 7, the parameters of $\mathrm{OMN}_{\mathrm{C}}$ and $\mathrm{OMN}_{\mathrm{P}}$ can be derived as

$$
\begin{aligned}
& \mathrm{a}=-R_{\mathrm{OPT}}\left(\frac{\alpha}{\alpha_{1}}(1+\gamma)-2\right) c /\left((1+\beta) X_{L} / 2\right) \\
& \mathrm{b}=R_{\mathrm{OPT}}(1+\beta) R_{L}\left(\left(\alpha / \alpha_{1}\right)(1+\gamma)-1\right) c \\
& \mathrm{c}=\sqrt{\frac{\left(2\left(\alpha / \alpha_{1}\right)(1+\gamma)-1\right)}{\left[R_{\mathrm{OPT}}(1+\beta) R_{L}\left(\left(\alpha / \alpha_{1}\right)(1+\gamma)-1\right)\left(\left(\alpha / \alpha_{1}\right)(1+\gamma)+1\right)\right]}} \\
& \mathrm{d}=\frac{R_{O P T} c(1+\beta) X_{L}\left(\left(\frac{\alpha}{\alpha_{1}}\right)(1+\gamma)-1\right)}{\left(2\left(\frac{\alpha}{\alpha_{1}}\right)(1+\gamma)-1\right)} \\
& a_{1}=\sqrt{\frac{R_{O P T}}{(1+1 / \beta) R_{L}}} \\
& b_{1}=-\sqrt{\left(1+\frac{1}{\beta}\right) R_{L} R_{O P T}}\left(1+\frac{1}{\beta}\right) X_{L} / 2 \\
& c_{1}=0 \\
& d_{1}=\sqrt{(1+1 / \beta) R_{L} / R_{O P T}}
\end{aligned}
$$

Using (23)-(30), the output networks parameters can be obtained and designed.

\section{Design of The Proposed DPA}

In the following, a closed process is presented to design a DPA with working frequencies of $1.2-2.8 \mathrm{GHz}$ based on the theories of Section III. To validate the presented means, a broadband high-efficiency DPA employing CGH40010F transistors is elaborated on Rogers R4350B substrate. The drain voltage $V_{\text {ds }}$ is $28 \mathrm{~V}$. The gate voltages $V_{\mathrm{gs}}$ for the carrier PA and the peak PA are $-2.8 \mathrm{~V}$ and $-6.0 \mathrm{~V}$, respectively. The optimum load impedance $R_{\mathrm{OPT}}$ is determined as $32 \Omega$ for the $\mathrm{CGH} 40010 \mathrm{~F}$ considering $V_{\text {knee. }}$. To obtain the optimized load impedance $Z_{\mathrm{OPT}}$ in the package plane, load-pull simulation is processed using the ADS software for deriving package parameters. From [20], the package parameters of CGH40010F can be obtained as shown in Fig. 8, which will be used in practical design process.

\section{A. Output Matching Network}

The design process of output network can be performed as follows. The $\mathrm{OBO}$ and desired bandwidth are determined firstly, where a $6 \mathrm{~dB}$ OBO and maximum relative bandwidth of $78 \%$ are chosen. Then, the coefficient $\gamma$, current ratio $\beta$ and $R^{*}$ are given by $\pm 0.74,1.16$ and $25 \Omega$ from Fig. 4 and 5, respectively. It is calculated that $\alpha, \alpha_{1}$ and $X_{\mathrm{L}}$ are 1.85, 2.37 and 0.76 using (20)-(22), respectively. The load impedance at the combine node, $Z_{\mathrm{L}}$, is fixed to be $15 \cdot(1 \pm j 0.76) \Omega$. The general transistor CGH40010F is used in this work. The ABCD transfer matrix of
$\mathrm{OMN}_{\mathrm{C}}$ and $\mathrm{OMN}_{\mathrm{P}}$ can be derived using (23)-(30). The designed $\mathrm{OMN}_{\mathrm{C}}$ and $\mathrm{OMN}_{\mathrm{P}}$ are shown in Fig. 8(a) by using network synthesis and optimization. Also, package parasitic parameters are absorbed in the output matching networks during optimizing the output matching circuits. In the analysis of Section III, the peak PA is assumed to be ideal. Of course, the actual peak PA may not be ideal. It may reduce the performance of Doherty. For Doherty PAs, the efficiency at the power back-off is more important. Especially at the power back-off, if the peak PA branch is not completely open, the power of the carrier PA would leak to the peak PA branch, resulting in reduced efficiency at the power back-off. Therefore, when optimizing the output network of the peak PA, the first thing is to ensure that the peak PA is in an open-circuit state at the power back-off. The simulated S11 of the designed $\mathrm{OMN}_{\mathrm{C}}$ and $\mathrm{OMN}_{\mathrm{P}}$ are shown in Fig. 8(b). It can be seen that the $\mathrm{S} 11$ of $\mathrm{OMN}_{\mathrm{C}}$ and $\mathrm{OMN}_{\mathrm{P}}$ is lower than $-10 \mathrm{~dB}$ in $1.2-2.8 \mathrm{GHz}$, which indicates the effectiveness of the matching circuits.

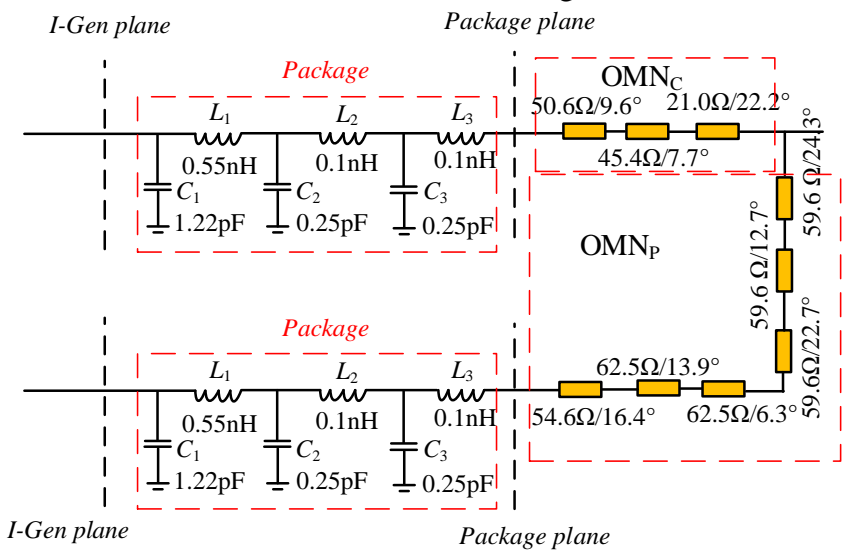

(a)

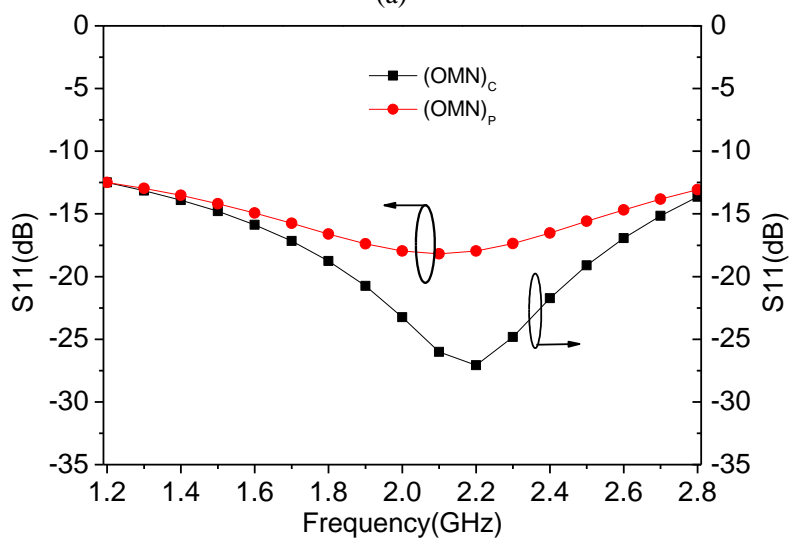

(b)

Fig. 8. Output matching networks with device parameters and simulated S11 (a) designed $\mathrm{OMN}_{\mathrm{C}}$ and $\mathrm{OMN}_{\mathrm{P}}(\mathrm{b})$ simulated $\mathrm{S} 11$.

\section{B. Post Matching Network}

The complete post matching circuit is shown in Fig. 9(a). Fig. 9(b) shows that the simulated input impedance is acceptable compared with the theoretical value of $Z_{\mathrm{L}}$. As mentioned before, the load impedance $Z_{\mathrm{L}}$ is $15 \bullet(1 \pm j 0.76) \Omega$. Post-matching circuits design aims to enable the load impedance to match the $50 \Omega$ standards while the harmonic impedance is properly controlled. As shown in Fig. 9, 
micro-strip lines TL1, TL2, TL3 and TL4 are used to adjust the harmonic impedances, which makes the second harmonic impedance close to zero and the third harmonic impedance approaching infinity. In addition, harmonic control circuit plays a role in the fundamental impedance transformation, and it can improve the efficiency of DPA. As we known, the harmonic networks would limit the bandwidth of PAs. It is noted that in this work, the harmonic networks are introduced after the combiner instead of in the carrier PA branch and peak PA branch, which is beneficial for broadband design.

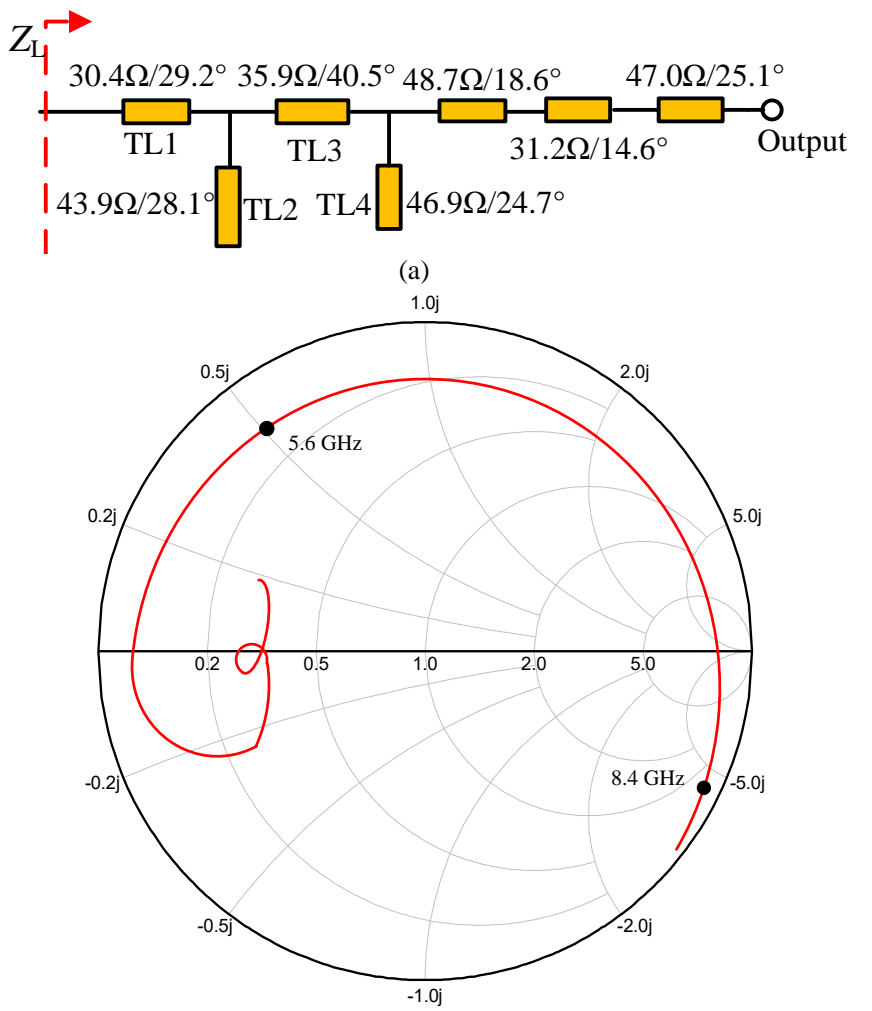

(b)

Fig. 9. (a) Post matching networks (b) Simulated impedance of the post matching networks (normalized $50 \Omega$ ).

\section{Input Matching Network and DPA Overall Circuit}

Stepped impedance topology is used to synthesize suitable input circuits, and provide a targeted saturation gain of around $10 \mathrm{~dB}$. A resistor $R$ is used to maintain the stability of DPAs. Before input matching networks of PAs, a three-stage Wilkinson divider is added to split the signal. In order to satisfy the bandwidth requirement, three stages are applied in the Wilkinson divider. Offset lines are also added in the input networks for ensuring that the signals' phases of the two branches are consistent at the combine node. After all networks are designed, these circuits are combined into a completed DPA. Of course, such a simple combination of DPA may not have the best performance, due to the mutual influence between different circuit networks, and/or non-ideal components. Optimized operation is necessary to optimize the performance of the DPA. The circuit of distributed parameters is finally optimized as shown in Fig. 10.

Fig .11(a) shows the drain efficiency versus output power in different target frequency bands by simulating the designed DPA circuits.

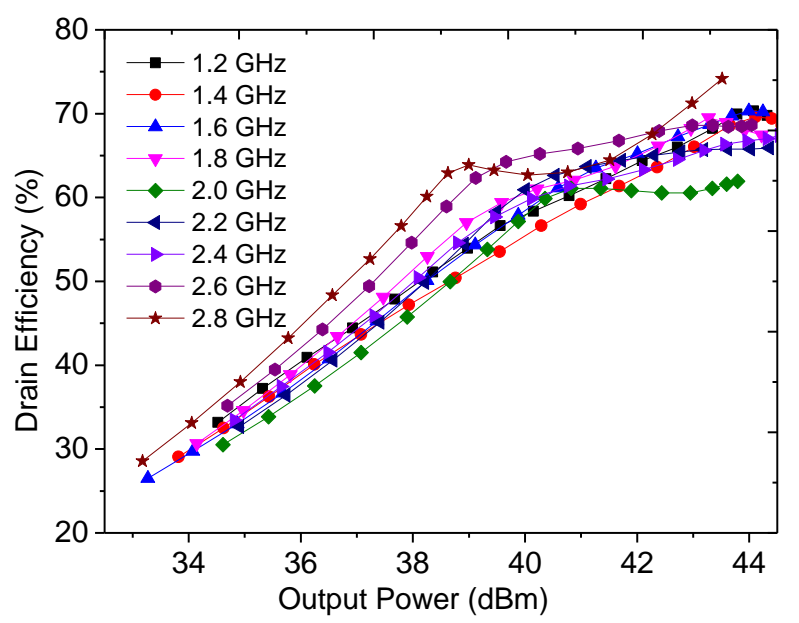

(a)

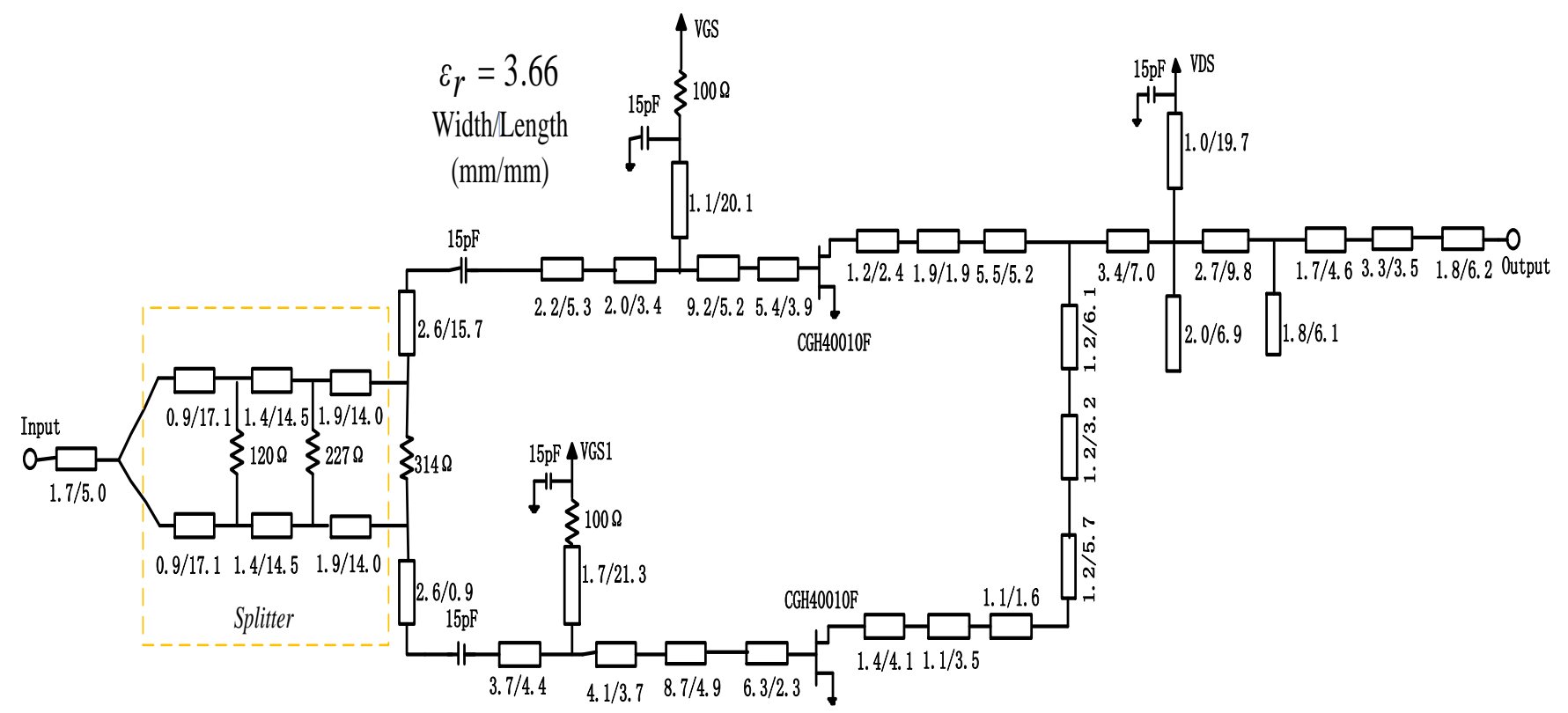

Fig. 10. Completed circuit schematic and parameters value. 


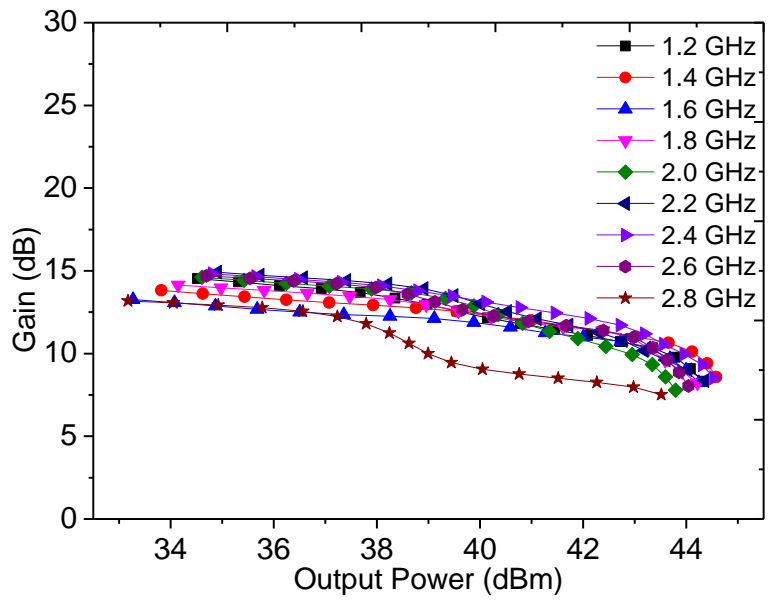

(b)

Fig. 11. Simulated performance of the designed DPA(a) drain efficiency, and (b) gain.

Seen from Fig. 11, the designed DPA can get over $43 \%$ drain efficiency at the $6 \mathrm{~dB}$ OBO level in 1.2-2.8 GHz. It verifies the effectiveness of the proposed method. Fig. 12 shows simulated impedance traces of the carrier PA at different frequencies. The green line is the simulated impedance at the saturation level, which is closer to the $R_{\mathrm{OPT}}$. It is consistent with the theorical value. The red line is the boundary line we calculated earlier, which roughly corresponds to $\gamma$ of $\pm 0.74, R^{*}$ of $25 \Omega$. Thus, the area on the right of the red line represents the theoretical values corresponding to the blue line in the figure. The dark red line represents the simulated impedance in the target frequency band at the OBO level, which roughly conforms to the theoretical value. The simulated load modulation trajectories of carrier PA and peak PA are shown in Fig. 13. Obviously, although there are some differences from the ideal conditions shown in Fig. 7, both the carrier and the peak PA can achieve load modulation well.

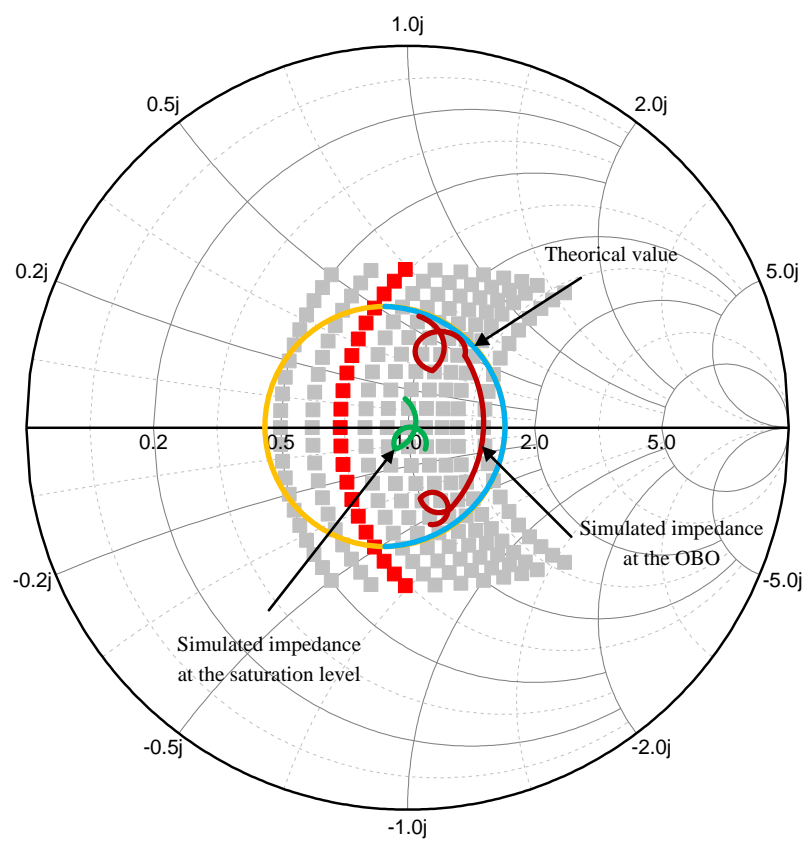

Fig. 12. Simulated load impedance of the carrier PA at the current plane.
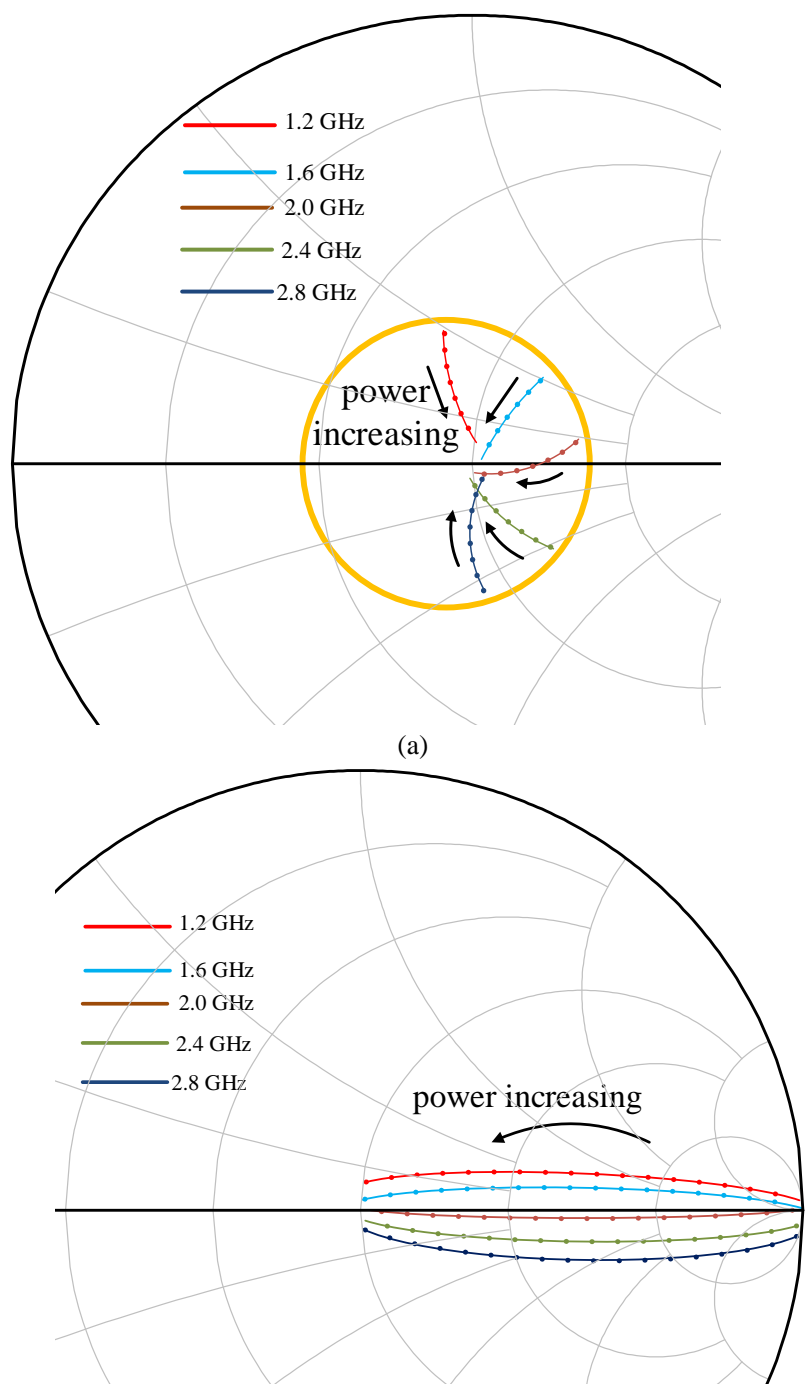

(b)

Fig. 13. Load modulation trajectories of carrier PA and peak PA. (a) carrier PA (b) peak PA.

\section{EXPERIMENT AND RESULTS ANALYSIS}

According to the circuits shown in Fig. 10, a DPA is fabricated by using Rogers R4350B $\left(\varepsilon_{\mathrm{r}}=3.66, H=30 \mathrm{mil}\right)$ substrate. Fig. 14 shows a photo of the implemented DPA.

Then, measurements are done including S-parameters and large signal performance. The simulated and measured S-parameters are shown in Fig. 15. In 1.2-2.8 GHz, the measured $\mathrm{S} 21$ is between $13.5 \mathrm{~dB}$ and $16 \mathrm{~dB}$. Correspondingly, the measured S11 is smaller than $-10 \mathrm{~dB}$.

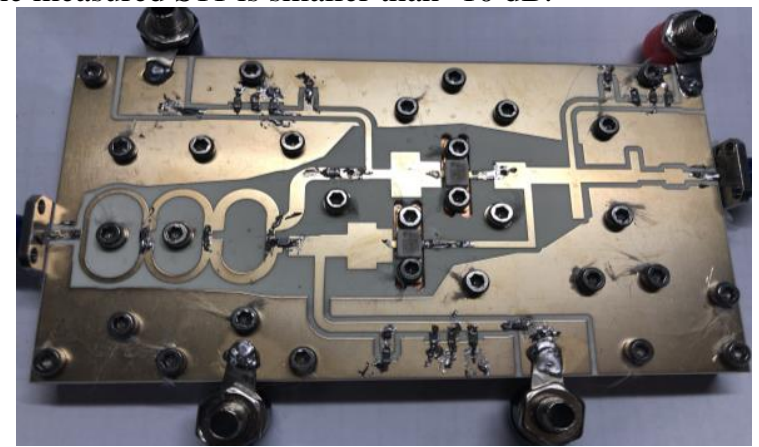

Fig. 14. Photo of the implemented DPA. 


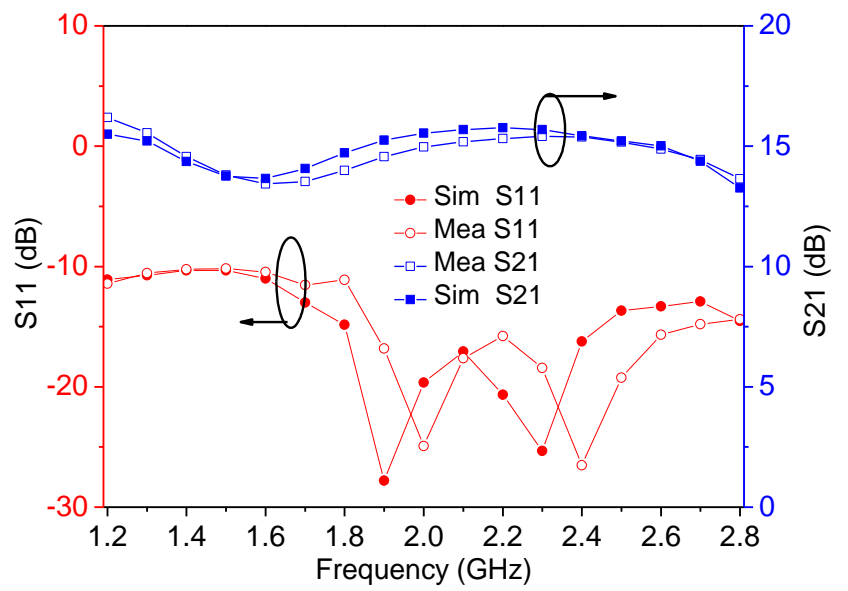

Fig. 15. Simulated and measured S11, S21 of the proposed DPA.

\section{A. Continuous Wave Testing}

Output power, drain efficiency and gain of the implemented DPA are measured by using continuous wave signals. The power of input signal is from $20 \mathrm{dBm}$ to $36 \mathrm{dBm}$.

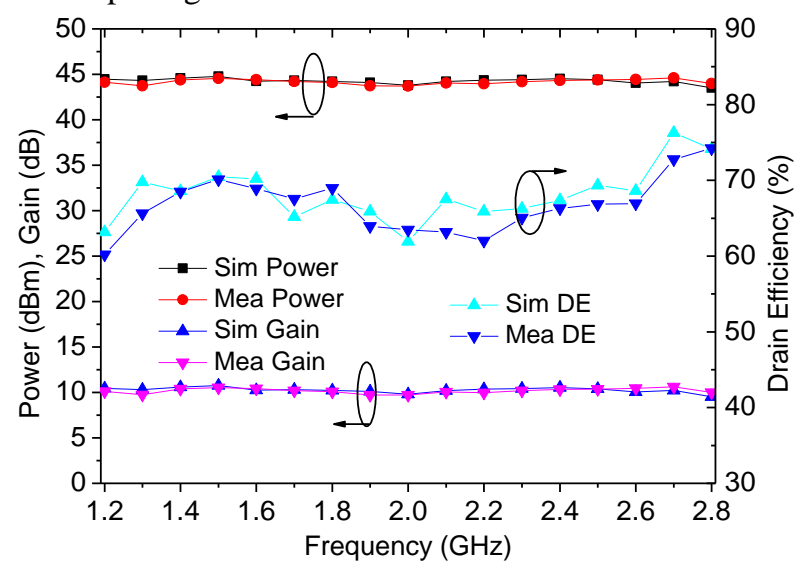

Fig. 16. Simulated and measured performance of the designed DPA at the saturation level.

Fig. 16 and 17 display the measured performances of the proposed DPA. As shown in Fig. 16, the DPA can deliver saturated output power of 43.7-44.1 dBm and saturated drain efficiency is from $60.5 \%$ to $74.2 \%$ in the frequency range of 1.2-2.8 GHz, while gain is between $9.7 \mathrm{~dB}$ and $10.5 \mathrm{~dB}$. From Fig. 17(a), the drain efficiency at the $6 \mathrm{~dB}$ OBO level is 48.1-57.6\%. As shown in Fig. 17(c), power added efficiency (PAE) can be achieved from $43.4 \%$ to $63.5 \%$ during load modulation in $1.2-2.8 \mathrm{GHz}$.

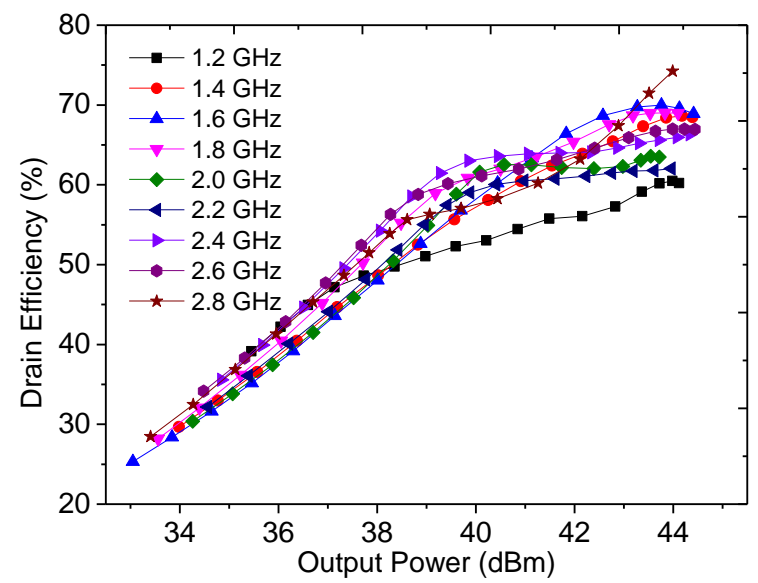

(a)

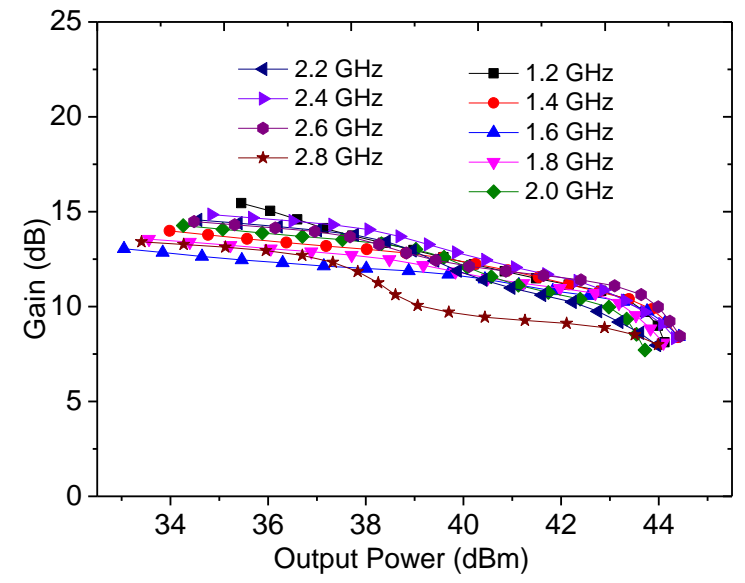

(b)

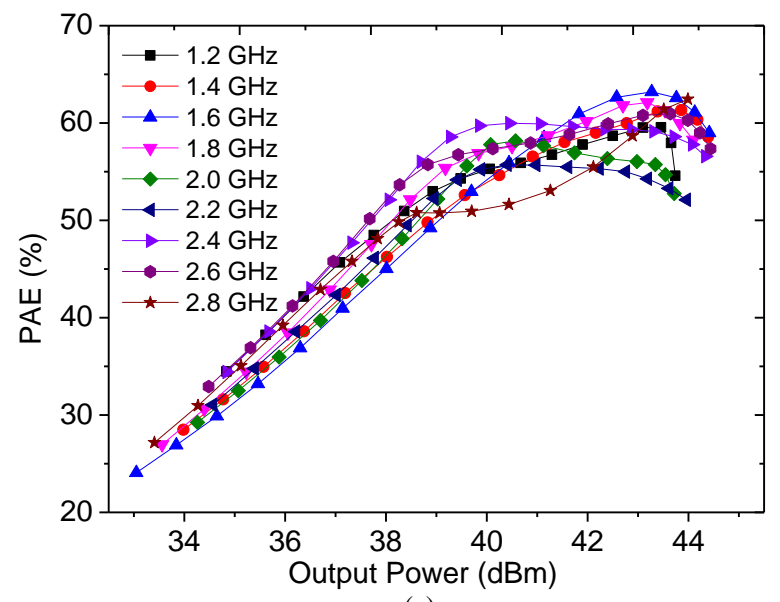

(c)

Fig. 17. Measured performances. (a) drain efficiency (b) gain and (c) PAE.

TABLE I

PERFORMANCES COMPARED WITH RECENT DPAS

\begin{tabular}{cccccc}
\hline \hline Ref. & $\begin{array}{c}\text { Freq (B.W.) } \\
(\mathrm{GHz})\end{array}$ & $\begin{array}{c}\text { Pout } @ \text { SAT } \\
(\mathrm{dBm})\end{array}$ & $\begin{array}{c}\text { DE@ SAT } \\
(\%)\end{array}$ & $\begin{array}{c}\text { DE@ 6dB } \\
\text { OBO }(\%)\end{array}$ & Device \\
\hline $2019[19]$ & $1.1-2.4(74 \%)$ & $43.3-45.4$ & $55-68$ & $43.8-54.9$ & $2 * 13 \mathrm{~W} \mathrm{GaN}$ \\
$2016[22]$ & $1.7-2.8(50 \%)$ & $44.5-46.3$ & $60-77$ & $52-66$ & $2 * 16 \mathrm{~W} \mathrm{GaN}$ \\
$2018[23]$ & $1.6-2.7(51 \%)$ & $43.8-45.2$ & $56-75.3$ & $46.5-63.5$ & $2 * 13 \mathrm{~W} \mathrm{GaN}$ \\
$2018[25]$ & $3.3-3.75(13 \%)$ & $48-48.5$ & $58-71$ & $47-59$ & $2 * 16 \mathrm{~W} \mathrm{GaN}$ \\
$2013[28]$ & $1.5-2.6(55 \%)$ & $41.5-45$ & $33-52$ & $29-49$ & $2 * 13 \mathrm{~W} \mathrm{GaN}$ \\
$2018[29]$ & $1.5-3.8(87 \%)$ & $42.3-43.4$ & $42-63$ & $33-55$ & $2 * 13 \mathrm{~W} \mathrm{GaN}$ \\
$2014[30]$ & $1.0-2.5(83 \%)$ & $40-42$ & $45-83$ & $35-58$ & $2 * 8 \mathrm{~W} \mathrm{GaN}$ \\
$2018[31]$ & $1.5-2.6(54 \%)$ & 41.8 & $40-45$ & $31-35$ & $2 * 13 \mathrm{~W} \mathrm{GaN}$ \\
$2019[32]$ & $1.2-2.4(67 \%)$ & $42-45$ & $41.6-81$ & $35-63$ & $2 * 13 \mathrm{~W} \mathrm{GaN}$ \\
This work & $1.2-2.8(78 \%)$ & $43.7-44.1$ & $60.5-74.2$ & $48.1-57.6$ & $2 * 13 \mathrm{~W} \mathrm{GaN}$ \\
\hline
\end{tabular}


Table I compares the measured drain efficiency and power between the proposed DPA and those in [19], [22-23], [25], [28-32]. It is shown that the fabrication bandwidth of the proposed one, $78 \%$, is larger than the others except the ones in [29] and [30]. Compared with [29] and [30], the drain efficiency in this work is higher than those of [29] and [30] at the saturation level and the OBO level. The proposed DPA is considered in terms of drain efficiency and bandwidth, which can provide a good performance balance and makes it more suitable for practical applications.

\section{B. $20 \mathrm{MHz}$ 6.5dB LTE Testing}

In order to characterize the linearity of the implemented DPA, the adjacent channel ratio (ACLR) is tested by using an LTE signal with a bandwidth of $20 \mathrm{MHz}$ and PAPR of $6.5 \mathrm{~dB}$. The measured ACLR with an average $37.5 \mathrm{dBm}$ output power is plotted in Fig. 18. It reveals that the ACLR is better than -27.4 $\mathrm{dBc}$ at $2 \mathrm{GHz}$. After adopting digital pre-distortion technology (DPD), the ACLR value is better than $-48.6 \mathrm{dBc}$.

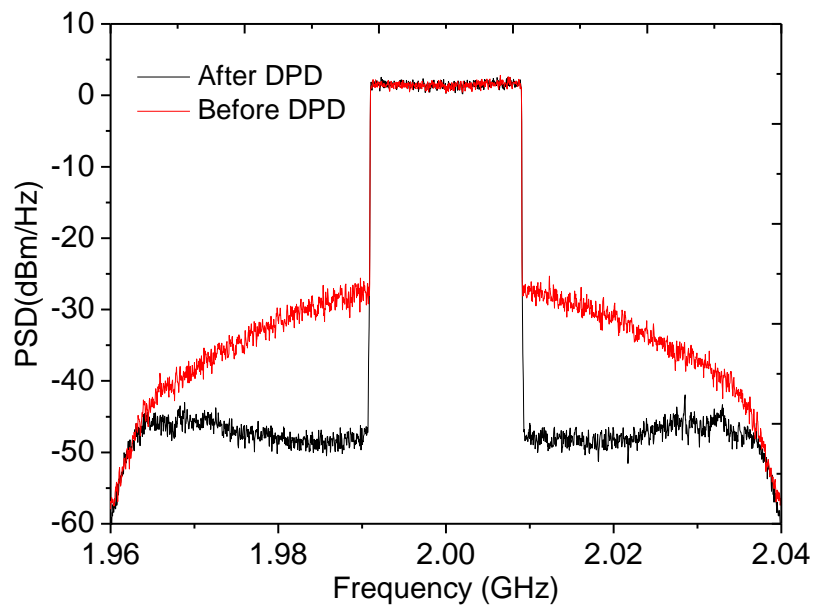

Fig. 18. Measured ACLR at $2.0 \mathrm{GHz}$ with a $37.5 \mathrm{dBm}$ average output power.

\section{CONCLUSION}

This paper applies the class-EFJ PA as the carrier PA of DPAs. Bandwidth characteristic of the DPAs with the class-EFJ PA is analyzed. Maximum relative bandwidth of $78 \%$ can be achieved by properly selecting corresponding parameter values. The design parameters are derived, and the design process is described in detail. For validation, a broadband DPA operating in $1.2-2.8 \mathrm{GHz}$ is designed and fabricated. Measurements show that the designed DPA based on the proposed theory and method can deliver over $43 \mathrm{dBm}$ output power with over $60 \%$ drain efficiency in saturation in 1.2-2.8 GHz. Moreover, at the $6 \mathrm{~dB}$ OBO stage, the drain efficiency can be higher than $48.1 \%$ in $1.2-2.8 \mathrm{GHz}$.

\section{REFERENCES}

[1] M. Li, J. Pang, Y. Li and A. Zhu, "Bandwidth Enhancement of Doherty Power Amplifier Using Modified Load Modulation Network," IEEE Trans. Circuits Syst. I, Reg. Papers., vol. 67, no. 6, pp. 1824-1834, Jun. 2020.

[2] X. Y. Zhou, S. Y. Zheng, W. S. Chan, S. Chen, and D. Ho, "Broadband efficiency-enhanced mutually coupled harmonic post-matching Doherty power amplifier," IEEE Trans. Circuits Syst. I, Reg. Papers., vol. 64, no. 7, pp. 1758-1771, Jul. 2017.

[3] S. Y. Zheng, Z. W. Liu, Y. M. Pan, Y. Wu, W. S. Chan, and Y. Liu, "Bandpass filtering Doherty power amplifier with enhanced efficiency and wideband harmonic suppression," IEEE Trans. Circuits Syst. I, Reg. Papers., vol. 63, no. 3, pp. 337-346, Mar. 2016.

[4] H. C. Chang, Y. Hahn, P. Roblin, and T. W. Barton, "New mixed mode design methodology for high-efficiency out-phasing chireix amplifiers," IEEE Trans. Circuits Syst. I, Reg. Papers., vol. 66, no. 4, pp. 1594-1607, Apr. 2019.

[5] D. F. Kimball et al., "High-efficiency envelope-tracking W-CDMA base station amplifier using GaN HFETs," IEEE Trans. Microw. Theory Techn., vol. 54, no. 11, pp. 3848-3856, Nov. 2006.

[6] K. Bathich, A. Z. Markos, and G. Boeck, "Frequency response analysis and bandwidth extension of the Doherty amplifier," IEEE Trans. Microw. Theory Techn., vol. 59, no. 4, pp. 934-944, Apr. 2011.

[7] X. Y. Zhou, S. Y. Zheng, W. S. Chan, S. Chen, and D. Ho, "Broadband efficiency-enhanced mutually coupled harmonic post-matching Doherty power amplifier," IEEE Trans. Circuits Syst. I, Reg. Papers., vol. 64, no. 7, pp. 1758-1771, Jul. 2017.

[8] R. Giofre, L. Piazzon, P. Colantonio, and F. Giannini, "A Doherty architecture with high feasibility and defined bandwidth behavior," IEEE Trans. Microw. Theory Techn., vol. 61, no. 9, pp. 3308-3317, Sep. 2013.

[9] D. Kang, D. Kim, Y. Cho, B. Park, J. Kim, and B. Kim, "Design of bandwidth-enhanced Doherty power amplifiers for handset applications," IEEE Trans. Microw. Theory Techn., vol. 59, no. 12, pp. 3474-3483, Dec. 2011.

[10] J. Xia, M. Yang, and A. Zhu, "Improved Doherty amplifier design with minimum phase delay in output matching network for wideband application," IEEE Microw. Wireless Compon. Lett., vol. 26, no. 11, pp. 52-54, Nov. 2016.

[11] J. M. Rubio, J. Fang, V. Camarchia, R. Quaglia, M. Pirola, and G. Ghione, "6-GHz wideband GaN Doherty power amplifier exploiting output compensation stages," IEEE Trans. Microw. Theory Techn., vol. 60, no. 8, pp. 2543-2548, Aug. 2012.

[12] J. Xia, M. Yang, Y. Guo, and A. Zhu, "A broadband high-efficiency Doherty power amplifier with integrated compensating reactance," IEEE Trans. Microw. Theory Techn., vol. 64, no. 7, pp. 2014-2024, Jul. 2016.

[13] J. Xia, W. Chen, F. Meng, C. Yu, and X. Zhu, "Improved three stage Doherty amplifier design with impedance compensation in load combiner for broadband applications," IEEE Trans. Microw. Theory Techn., vol. 67, no. 2, pp. 778-786, Feb. 2019.

[14] J. Pang, S. He, C. Huang, Z. Dai, J. Peng, and F. You, “A post matching Doherty power amplifier employing low-order impedance inverters for broadband applications," IEEE Trans. Microw. Theory Techn., vol. 63, no. 12, pp. 4061-4071, Dec. 2015.

[15] X. Y. Zhou, S. Y. Zheng, W. S. Chan, X. Fang, and D. Ho, "Postmatching Doherty power amplifier with extended back-off range based on selfgenerated harmonic injection," IEEE Trans. Microw. Theory Techn., vol. 66, no. 4, pp. 1951-1963, Apr. 2018.

[16] J. Pang, S. He, Z. Dai, C. Huang, J. Peng, and F. You, "Design of a post matching asymmetric Doherty power amplifier for broadband applications," IEEE Microw. Wireless Compon. Lett., vol. 26, no. 1, pp. 52-54, Jan. 2016.

[17] H. Kang et al., "Octave bandwidth Doherty power amplifier using multiple resonance circuit for the peaking amplifier," IEEE Trans. Circuits Syst. I, Reg. Papers, vol. 66, no. 2, pp. 583-593, Feb. 2019.

[18] X.-H. Fang, H.-Y. Liu, K.-K.-M. Cheng, and S. Boumaiza, "Modified Doherty amplifier with extended bandwidth and back-off power 
range using optimized peak combining current ratio," IEEE Trans. Microw. Theory Techn., vol. 66, no. 12, pp. 5347-5357, Dec. 2018.

[19] Z. Yang et al., "Bandwidth extension of Doherty power amplifier using complex combining load with noninfinity peaking impedance," IEEE Trans. Microw. Theory Techn., vol. 67, no. 2, pp. 765-777, Feb. 2019.

[20] M. J. Pelk, W. C. E. Neo, J. R. Gajadharsing, R. S. Pengelly, and L. C. N. D. Vreede, "A high-efficiency $100-\mathrm{W}$ GaN three-way Doherty amplifier for base-station applications," IEEE Trans. Microw. Theory Techn., vol. 56, no. 7, pp. 1582-1591, Jul. 2008.

[21] Y. S. Lee, M. W. Lee, S. H. Kam, and Y. H. Jeong, "A highly linear and efficient three-way Doherty amplifier using two-stage GaN HEMT cells for repeater systems," Microw. Opt. Tech. Lett., vol. 51, no. 12, pp. 2895-2898, Dec. 2009.

[22] J. Kim, J. Cha, I. Kim, and B. Kim, "Optimum operation of asymmetrical-cells-based linear Doherty power amplifiers-uneven power drive and power matching," IEEE Trans. Microw. Theory Techn., vol. 53, no. 5, pp. 1802-1809, May 2005.

[23] X. Chen, W. Chen, F. M. Ghannouchi, Z. Feng, and Y. Liu, "A broadband Doherty power amplifier based on continuous-mode technology," IEEE Trans. Microw. Theory Techn., vol. 64, no. 12, pp. 4505-4517, Dec. 2016.

[24] W. Shi et al., "Broadband continuous-mode Doherty power amplifiers with noninfinity peaking impedance," IEEE Trans. Microw. Theory Techn., vol. 66, no. 2, pp. 1034-1046, Feb. 2018.

[25] C. Huang, S. He, and F. You, "Design of broadband modified class-J Doherty power amplifier with specific second harmonic terminations," IEEE Access, vol. 6, pp. 2531-2540, 2018.

[26] Z. Zhang, Z. Cheng, H. Ke, G. Liu and S. Li, "Design of a Broadband High-Efficiency Hybrid Class-EFJ Power Amplifier," IEEE Microw. Wireless Compon. Lett., vol. 30, no. 4, pp. 407-409, Apr. 2020.

[27] X. Fang, H. Liu, K. M. Cheng and S. Boumaiza, "Two-Way Doherty Power Amplifier Efficiency Enhancement by Incorporating Transistors' Nonlinear Phase Distortion," IEEE Microw. Wireless Compon. Lett., vol. 28, no. 2, pp. 168-170, Feb. 2018.

[28] X. A. Nghiem, J. Guan, T. Hone, and R. Negra, "Design of concurrent multiband Doherty power amplifiers for wireless applications," IEEE Trans. Microw. Theory Techn., vol. 61, no. 12, pp. 4559-4568, Dec. 2013.

[29] J. J. Moreno Rubio, V. Camarchia, M. Pirola, and R. Quaglia, "Design of an $87 \%$ fractional bandwidth Doherty power amplifier supported by a simplified bandwidth estimation method," IEEE Trans. Microw. Theory Techn., vol. 66, no. 3, pp. 1319-1327, Mar. 2018.

[30] R. Giofre, L. Piazzon, P. Colantonio, and F. Giannini, "A closed-form design technique for ultra-Wideband Doherty power amplifiers," IEEE Trans. Microw. Theory Tech., vol. 62, no. 12, pp. 3414-3424, Dec. 2014.

[31] M. S. Khan et al., "A novel two-stage broadband Doherty power amplifier for wireless applications," IEEE Microw. Wireless Compon. Lett., vol. 28, no. 1, pp. 40-42, Jan. 2018.

[32] G. Naah and R. Giofrè, "Empowering the Bandwidth of Continuous mode Symmetrical Doherty Amplifiers by Leveraging on Fuzzy Logic Techniques," IEEE Trans. Microw. Theory Techn., Techn., vol. 68, no. 7, pp. 3134-3147, Dec. 2020.

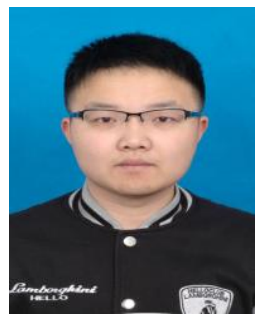

ZHIWEI ZHANG received the B.S. degree in electronic science and technology from Hangzhou Dianzi University, Hangzhou, China, in 2017, where he is currently pursuing the Ph.D. degree with Key Lab. of RF Circuit and System, Education Ministry. His current research interests include highly linear and efficient microwave PA design.

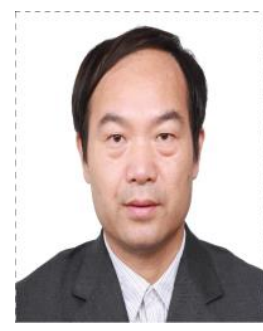

ZHIQUN CHENG received the B.S. and M.S. degrees from the Hefei University of Technology, Hefei, China, in 1986 and 1995, respectively, and the $\mathrm{Ph} . \mathrm{D}$. degree in microelectronics and solid state electronics from the Shanghai Institute of Metallurgy, Chinese Academy of Sciences, Shanghai, China, in 2000. From 1986 to 1997, he was a Teaching Assistant and a Lecturer with the Hefei University of Technology, China. From 2000 to 2005, he was an Associate Professor with the Shanghai Institute of Metallurgy, China. He is currently a Professor and the Dean of the School of Electronic and Information, Hangzhou Dianzi University. He has authored or co-authored over 150 technical journal and conference papers. His research interests include microwave theory and technology, MMIC, power amplifier, and RF front end. He is currently a member of a Council of Zhejiang Electronic Society. He was also a Chair of the Organizational Committee for over 10 International Conferences.

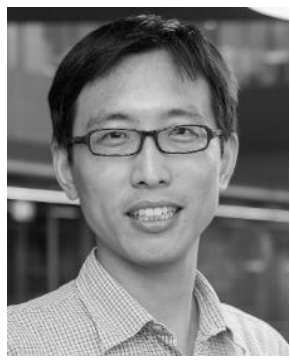

HANG LI received the B.Eng. and M.Eng. degrees from Beijing Jiaotong University, Beijing, China, in 2003 and 2006, respectively, and the Ph.D. degree from the University of Western Australia, Perth, WA, Australia, in 2014, all in electronic engineering. From 2016 to 2019, he was a Postdoctoral Research Fellow with the Global Big Data Technologies Centre, University of Technology Sydney, Sydney, NSW, Australia. He is currently with the College of Electronics and Information, Hangzhou Dianzi University, Hangzhou, China. His research interests include wireless and optical communications signal processing, wireless cross-layer protocols design, and mm-Wave Massive MIMO. 


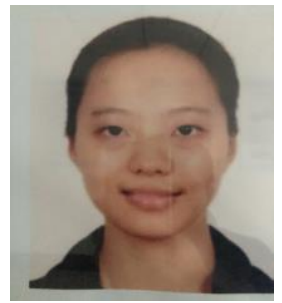

HUAJIE KE was born in Jiangsu, China in 1983. She received the B.S. degree in physics from Nanjing University, Nanjing, China, in 2006, the M.S. degree in physics from University of Massachusetts, USA, in 2009 and Ph.D. degree in physics from University of Massachusetts, USA, in 2013. Her research interest includes theoretical analysis, micro-magnetic simulation to material fabrication and characterization, especially on the overlap between conventional RF circuits and new artificial electromagnetic materials.

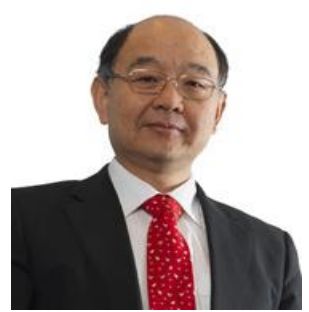

Y. Jay Guo (Fellow'2014) received a Bachelor Degree and a Master Degree from Xidian University in 1982 and 1984, respectively, and a PhD Degree from Xian Jiaotong University in 1987, all in China. His research interest includes antennas, mm-wave and $\mathrm{THz}$ communications and sensing systems as well as big data technologies. He has published over 470 research papers including 250 journal papers, most of which are in IEEE Transactions, and he holds 26 patents. He is a Fellow of the Australian Academy of Engineering and Technology, a Fellow of IEEE and a Fellow of IET, and was a member of the College of Experts of Australian Research Council (ARC, 2016-2018). He has won a number of most prestigious Australian Engineering Excellence Awards $(2007,2012)$ and CSIRO Chairman's Medal $(2007,2012)$, and was named one of the most influential engineers in Australia in 2014 and 2015, respectively.
Prof Guo is a Distinguished Professor and the Director of Global Big Data Technologies Centre (GBDTC) at the University of Technology Sydney (UTS), Australia. Prior to this appointment in 2014, he served as a Director in CSIRO for over nine years. Before joining CSIRO, he held various senior technology leadership positions in Fujitsu, Siemens and NEC in the U.K.

Prof Guo has chaired numerous international conferences and served as guest editors for a number of IEEE publications. He is the Chair of International Steering Committee, International Symposium on Antennas and Propagation (ISAP). He was the International Advisory Committee Chair of IEEE VTC2017, General Chair of ISAP2022, ISAP2015, iWAT2014 and WPMC'2014, and TPC Chair of 2010 IEEE WCNC, and 2012 and 2007 IEEE ISCIT. He served as Guest Editor of special issues on "Antennas for Satellite Communications" and "Antennas and Propagation Aspects of $60-90 \mathrm{GHz}$ Wireless Communications," both in IEEE Transactions on Antennas and Propagation, Special Issue on "Communications Challenges and Dynamics for Unmanned Autonomous Vehicles," IEEE Journal on Selected Areas in Communications (JSAC), and Special Issue on "5G for Mission Critical Machine Communications”, IEEE Network Magazine. 